\title{
La révolution pastorienne et les théories sur l'étiologie du goitre et du crétinisme*
}

par Bernardino Fantini

\section{RÉSUMé}

Tout au long du XIX e siècle un grand nombre de théories étiologiques différentes et contradictoires sur le goitre et le crétinisme avait été proposé. La reconnaissance par le corps scientifique et médical du poids de la théorie microbienne des maladies infectieuses poussa à chercher un agent microbien pour tous les états pathologiques et on chercha longtemps un microbe responsable du goitre endémique. Cette attitude, associé à l'absence d'une théorie générale des maladies de carence, qui ne sera élaborée qu'au début du $X X^{e}$ siècle, résulta en un retard important dans les connaissances sur l'étiologie du goitre et dl: crétinisme. Toutefois, le développement de la théorie des germes a déterminé des effets positifs, en particulier la diffusion d'un modèle scientifique nouveau et la confiance dans la possibilité de lutter efficacement contre les endémies par des mesures simples de prophylaxie généralisée.

\section{Introduction}

En 1889, au moment où se réunit à Paris le Congrès international d'hygiène, Paul Brouardel, président du Comité national consultatif d'hygiène publique et rédacteur des Annales d'hygiène publique et de médecine légale, exprime le respect et la reconnaissance de la profession médicale pour l'œuvre accomplie par Pasteur:

«Si les échos de cette séance arrivaient jusqu'à eux [...] nos ancêtres apprendraient que la plus formidable des révolutions qui, depuis trente siècles, ait secoué jusque dans ses fondements la science médicale, est l'œuvre d'un homme étranger à la corporation [...]. Tous, nous nous proclamons les disciples de Pasteur ${ }^{1} . »$

L'adhésion de l'ensemble du corps médical à la théorie microbienne des maladies infectieuses est rapide, et on la considère comme acquise quand le

* Communication présentée à la réunion de la Société suisse d'histoire de la médecine et des sciences naturelles, le 5 octobre 1990, à Genève.

Gesnerus 49 (1992) 21-38 
Congrès international d'hygiène de Budapest en 1894 accueille d'une façon triomphale l'annonce, par Emile Roux, de la mise au point d'un sérum contre la diphtérie. Après le succès de l'expérience de vaccination anticharbonneuse de Poully-le-Fort et les premiers traitements antirabiques, la sérothérapie antidiphtérique marque un lieu de passage symbolique: pour la première fois, la médecine avait à sa disposition une arme vraiment efficace contre une maladie infectieuse terrible, considérée à l'époque comme la maladie épidémique la plus meurtrière. On peut désormais se poser clairement pour objectif l'arrêt, voire l'élimination de certaines épidémies et endémies.

Il est vrai qu'à cette époque les hygiénistes sont à la pointe du mouvement de diffusion de la nouvelle doctrine et que les cliniciens sont du moins prudents sinon clairement opposés à une généralisation hâtive de l'étiologie bactérienne à toutes les maladies, en soulignant les manifestations cliniques particulières des maladies ${ }^{2}$. Mais dans l'ensemble, le milieu médical est contraint à considérer comme acquise la méthode pastorienne et à accepter les résultats tangibles qu'elle a rendus possibles ${ }^{3}$.

L'essor de la microbiologie produit à la fois une révolution théorique (la théorie des germes à laquelle est associée la technique d'atténuation de la virulence des microorganismes pathogènes, le développement de l'immunologie et de la sérothérapie) et une révolution pratique, dans l'hygiène et dans la thérapie. Les deux révolutions vont changer la médecine et surtout le rôle du médecin dans la société, grâce à une «médicalisation de la société», opérée par la législation de santé publique et la prophylaxie généralisée des maladies endémiques et épidémiques.

Ce processus a été très bien étudié par les historiens des sciences et de la médecine et il n'est évidemment pas nécessaire d'insister ici sur ce point. Il est toutefois important de noter que la "pastorisation de la médecine», comme l'a appelée Claire Salomon-Bayet ${ }^{4}$, change les acteurs de la médecine et surtout les rapports entre les différents acteurs.

Jusqu'à l'essor de la bactériologie, les hygiénistes s'intéressaient de préférence à des facteurs externes à l'individu, comme le climat, la terre, l'eau, l'air, les aliments. Les cliniciens au contraire s'intéressaient à des facteurs individuels, telles les constitutions et les humeurs. Enfin, la «médecine scientifique», la pathologie cellulaire, la chimie physiologique et la physiologie expérimentale étudiaient les facteurs internes de l'organisation vitale, en redéfinissant les rapports entre le normal et le pathologique, sans avoir un impact thérapeutique réel. Entre ces trois niveaux il n'y avait aucun 
contact et les découvertes dans un domaine n'étaitent pas nécessairement reportées dans les autres. Le pasteurisme unifia le travail ainsi que les résultats de ces trois groupes et permit d'établir un lien immédiat entre les conditions de vie, la diffusion des agents morbides et l'état de santé d'une population. Cela permit, simultanément, à l'hygiéniste de bien cibler ses objectifs et ses conseils, et au clinicien d'établir une étiologie spécifique et une thérapie efficace.

Le développement de la microbiologie transforme l'action du médecin, de l'hygiéniste et celle du législateur; on passe de l'amalgame des causes à la certitude de l'action, et la réalisation d'une politique sanitaire à grande échelle devient possible. La seule identification des agents pathogènes et des causes spécifiques, que l'on peut suivre dans leurs changements apparemment désordonnés, substitue à l'impuissance la capacité d'action, au découragement la confiance, au mélange de conseils vagues des indications prophylactiques et thérapeutiques précises. La simple définition d'un nouvel acteur réordonne les problèmes épidémiologiques qui semblaient défier toute analyse causale; elle suffit à rendre crédibles des programmes d'intervention nouveaux et ambitieux. Le concept de microbe établit ainsi un principe causal, suffisant ou seulement nécessaire, mais aussi un moyen d'action, indique des lieux et des déplacements, des conditions et des possibilités d'intervention. La connaissance des causes permet de prédire; elle concrétise également les programmes de longue haleine. C'est dans ce contexte que naissent les politiques de contrôle et d'éradication des maladies épidémiques, à travers la mise en place de législations adéquates, de protocoles internationaux et de mesures de prophylaxie.

Le cadre professionnel et institutionnel de la médecine et de la santé se trouve rapidement changé et une unification nécessaire, de lieu et de pratiques, entre recherche biologique, pratiques médicales et structures politiques et sociales se trouve d'emblée réalisée. Cette synthèse produit des conséquences qui vont bien au-delà du domaine des maladies infectieuses, pour influencer des domaines apparemment lointains, comme en l'occurence les études sur le goitre et le crétinisme endémiques.

\section{Les connaissances sur l'endémie du goitre}

Le goitre endémique et le crétinisme ainsi que leur distribution géographique, en particulier leur diffusion dans les Alpes, étaient connus depuis l'âge 
classique. Toutefois, leur histoire moderne peut commencer dès les premiers rapports systématiques sur la distribution géographique dans les Alpes et les Pyrénées, sur leur étiologie générale et prochaine, sur la thérapeutique individuelle et collective des affections thyrö̈diennes. Après les importantes recherches de la fin du XVIII ${ }^{\mathrm{e}}$ siècle ${ }^{5}$, une série importante de recherches biologiques, cliniques et épidémiologiques, menées tout au long du XIX ${ }^{\mathrm{e}}$ siècle, avaient produit un remarquable corpus de connaissances et un grand nombre de théories étiologiques différentes et même contradictoires ${ }^{6}$. A cette époque, les théories étiologiques oscillaient entre cinq pôles: 1) malfonctionnement physiologique, malformation anatomique, déséquilibre, disharmonie; 2) hérédité; 3) carences alimentaires;4) intoxication;5) contagion, maladie infectieuse.

Toutes ces causes semblaient être possibles et pour chacune d'entre elles on pouvait apporter de nombreux arguments contraires. Il ne suffit pas d'avoir des connaissances (par exemple l'effet positif de l'iode); il faut que ces connaissances soient placées à l'intérieur d'un cadre théorique cohérent et assez général. Par conséquent, le goitre est peut-être la maladie pour laquelle on a proposé le nombre le plus élevé de théories étiologiques, sans avoir la possibilité d'en préciser une qui serait spécifique et sur laquelle on concentrerait les efforts thérapeutiques et prophylactiques. La thérapie est, elle aussi, multiple et comprend l'intervention chirurgicale ${ }^{7}$, les conseils hygiéniques ou prophylactiques et les remèdes empiriques. Il n'y avait qu'un seul fait thérapeutique qui pouvait être considéré comme certain, consistant en l'efficacité de certains remèdes, telle l'éponge brûlée dans laquelle, à partir de la première moitié du XIX $\mathrm{X}^{\mathrm{e}}$ siècle, on peut démontrer la présence d'iode.

Jean-François Coindet (1774-1834), médecin à Genève, s'était fortement intéressé, pour des raisons évidentes, au traitement du goitre ${ }^{8}$. Dans une lettre écrite à Andrew Ure et publiée par celui-ci dans son Dictionnaire de chimie $^{9}$, Coindet donne un récit de sa découverte:

«Il y a deux ans que je cherchai dans le formulaire de Cadet de Gassicourt une formule qui fût connue à Paris et que je pusse indiquer à une dame de cette ville qui me consultait pour un goitre. J'y trouvai que Russel conseillait du fucus brûlé. Je soupçonnais que le principe commun entre l'éponge, dont nous nous servons avec succès ici contre le goitre, et le fucus, dont j'ignorais les propriétés, pourrait bien être l'iode: je l'essayai, avec infiniment de précautions, et je réussis [...] Une grande pratique m'avait fourni, pendant une année entière, un grand nombre et une variété infinie de cas: ma découverte faisait du bruit.»

Ce bruit était en large partie justifié, car les résultats n'étaient pas toujours bons et l'usage de l'iode pouvait avoir aussi de sérieuses conséquences 
négatives. Plusieurs échecs furent signalés et soulevèrent un débat assez animé, surtout en Suisse, car les médecins suisses connaissaient les effets négatifs de l'usage de l'iode. Coindet lui-même avait attiré l'attention sur des phénomènes de «saturation», sur un «point médical» au-delà duquel l'effet du remède détermine des symptômes typiques («action constitutionnelle de l'iode») ${ }^{10}$. Par conséquent, comme l'écrit Coindet, «il ne s'agit donc pas de dire: vous avez le goitre, prenez de l'iode». Toutefois, ces échecs et ces précautions limitèrent la portée théorique de la découverte de Coindet, jusqu'au scepticisme.

Après la première proposition émise par Boussingault ${ }^{11}$, les recherches sur la relation étiologique entre la teneur en iode des eaux potables et la distribution géographique du goitre endémique et du crétinisme avaient déjà entraîné des expériences de prophylaxie collective de l'hypothyroidisme par l'ioduration de l'eau ou du sel de cuisine. Citons à ce propos les travaux de J.-L.Prévost (1790-1850) à Genève ainsi que de J.-J. Grange (1819-1892) ${ }^{12}$ et de G. A. Chatin (1813-1901) en France. Gaspard Adolphe Chatin, botaniste et chimiste, directeur de l'Ecole Supérieure de Pharmacie de Paris, étudia la distribution de l'iode dans la nature et proposa en 1850 que la carence d'iode dans l'eau potable pouvait bien être «la cause principale du goitre» ${ }^{13}$. L'Académie des Sciences organisa une enquête pour vérifier cette affirmation, mais les résultats ne furent pas favorables à Chatin, car la distribution géographique du goitre et du crétinisme n'était pas parallèle à la pauvreté en iode des eaux.

\section{La microbiologie comme obstacle épistémologique et stimulus}

L'idée que le goitre et le crétinisme pouvaient être associés à une contagion, et donc être des maladies infectieuses, avait été proposée en plusieurs occasions. Une raison pour attribuer une grande importance à la théorie contagionniste du goitre et du crétinisme était que la diffusion de la maladie dans les villages et les régions géographiques se montrait identique à la distribution typique d'une maladie infectieuse à diffusion lente. A la fin du $\mathrm{XIX}^{\mathrm{e}}$ siècle, la reconnaissance par le corps scientifique et médical du poids de la théorie microbienne des maladies infectieuses poussa à chercher à tout prix un agent microbien pour tous les états pathologiques de sorte qu'on rechercha longtemps un microbe responsable du goitre et du crétinisme endémiques. Cette attitude, associée à l'absence d'une théorie générale des maladies de carence, qui ne sera élaborée qu'au début du $\mathrm{XX}^{\mathrm{e}}$ siècle, 
entraîna un retard important dans les connaissances sur l'étiologie de ces pathologies. Les différentes théories sur l'étiologie du goitre (l'iode, le terrain, l'eau, etc.) étaient considérées par les bactériologistes, de même que par les pathologistes cellulaires, comme le résidu d'une médecine dépassée, la médecine des constitutions et des dispositions.

Par exemple, Rudolf Virchow, dans le cadre de son analyse des processus de croissance pathologique, considère le goitre comme une «formation active et progressive, provoquée par une irritation». Ce processus irritatif ne pouvait pas être le résultat d'une carence, mais au contraire d'une substance active. Après avoir parlé du rôle possible de la carence d'iode dans l'eau, il formule la conclusion que:

«il est très difficile de croire qu'un processus actif, en particulier irritatif, puisse être déterminé seulement par une déficience (Mangel), plutôt que par une substance ou une combinaison positive» ${ }^{14}$.

Par ailleurs, la même situation se produit aussi pour les «maladies chimiques», comme le scorbut et le beri-beri, et on peut établir un parallélisme très intéressant et très instructif entre ces deux domaines médicaux. Le succès de la bactériologie et de la pathologie cellulaire fut pour les maladies de carence un obstacle épistémologique ${ }^{15}$, produit par l'asymétrie entre une causalité positive et une causalité négative : il est plus facile d'émettre une hypothèse à partir d'une cause externe, d'une "présence», plutôt que d'imaginer une carence d'un facteur essentiel, une absence d'un quid qui est indispensable ${ }^{16}$. En plus, comme le savent bien les philosophes, une absence ne constitue pas une preuve définitive. L'étiologie à la fin du XIX ${ }^{\mathrm{e}}$ siècle dans le cadre d'une vision plutôt mécaniste de l'organisation vitale est dominée par les causes positives, c'est-à-dire les agents morbides. C'est seulement après le développement de l'immunologie et de l'endocrinologie qu'une vision intégrée de l'organisme va donner aux causes négatives, à l'absence de facteurs indispensables, un rôle causal des pathologies.

\section{Les recherches sur le goitre de G. B. Grassi}

Après l'origine et les grands succès de la bactériologie, on assiste dans les pays intéressés par le goitre et le crétinisme, et tout particulièrement en Suisse et en Italie, au développement d'une série de recherches visant à isoler l'agent causal de la maladie. J'ai étudié en détail les recherches sur le goitre conduites en Italie par Giovanni Battista Grassi et son collaborateur L. Mu- 
naron, et en particulier les notes de laboratoire conservées à l'Institut d'anatomie comparée de l'Université de Rome «La Sapienza», lesquelles concernaient une série d'expériences visant à découvrir l'agent étiologique de la maladie ${ }^{17}$.

Les résultats impressionnants obtenus par Grassi et ses collègues de Rome sur le vecteur du paludisme avaient poussé Grassi à imaginer qu'une méthode similaire pouvait être appliquée avec succès aux autres endémies graves qui frappaient l'Italie à cette époque, et tout particulièrement le goitre, le crétinisme et la pellagre ${ }^{18}$.

Cette méthode, une combinaison de biogéographie et d'épidémiologie, associée au contrôle continu en laboratoire, consistait en l'analyse ponctuelle des variations dans le degré d'endémie entre les différentes régions, afin d'isoler une variation corrélative d'un ou de plusieurs paramètres. Cette méthode avait permis à Grassi d'identifier dans la présence d'espèces déterminées du genre Anopheles le facteur spécifique lié à la transmission du paludisme. Un autre exemple de l'application de la même méthode est la découverte, par Grassi, du facteur causal de la nécrose phosphorique liée à l'usage du phosphore blanc dans les usines de fabrication d'allumettes, à la suite d'une enquête générale menée sur l'ensemble du territoire italien avec une analyse détaillée de différentes conditions de production et de travail.

En septembre 1902, Grassi et Munaron commencent leur recherche, essayant de montrer le "pouvoir goitrogène» de certaines eaux; ils arrivent rapidement à montrer l'existence d'«eaux goitrogènes sans goitre»-expression qui fait significativement pendant avec celle, plus célèbre, de «anophélisme sans malaria» - car les personnes qui séjournaient dans des régions à forte endémicité de goitre ne contractaient pas la maladie.

Grassi et Munaron réalisent alors à Cogne, dans la Vallée d'Aoste, un village à très forte endémicité, des expériences sur des chiens provenant de différentes localités où le goitre n'était pas endémique. Tous les chiens, après une période variable, développaient le goitre, même avec un régime contrôlé et l'usage d'eau bouillie. Sur la base des résultats obtenus avec de la terre humide, Grassi et Munaron échafaudent l'hypothèse du rôle pathogénique des terrains humides et non simplement de l'eau en tant que telle; puisque, lors d'autres expériences, des chiens élevés dans des cages soulevées, sans contact avec le terrain, devenaient également goitreux, le facteur causal de la maladie devait donc être transporté par l'air.

Une expérience témoin fut réalisée à Rome, dans le laboratoire de Grassi. De l'eau, de la terre mouillée provenant de Cogne furent utilisées pour 
«infecter» des chiens et démontrer leur capacité à provoquer la formation du goitre, sans aucun succès. Les deux chercheurs essayèrent aussi de prouver l'hypothèse de la contagion directe, en utilisant des extraits thyroïdiens et du sang de chiens goitreux. Par un contrôle attentif des conditions de laboratoire ils arriveront rapidement à exclure la présence éventuelle de vecteurs de l'infection.

Cette série d'expériences se solda donc par un véritable échec. La difficulté principale à laquelle se heurta Grassi était la même que celle que rencontraient les médecins du $\mathrm{XIX}^{\mathrm{e}}$ siècle, c'est-à-dire le déterminisme multifactoriel de l'endémie qui ne permettait pas d'isoler un seul facteur étiologique. Il y avait, en plus, une difficulté d'ordre pratique, car l'enquête très étendue que Grassi considérait indispensable nécessitait des ressources humaines et financières beaucoup plus importantes que celles dont il disposait. Cette recherche était donc au-delà des possibilités de son laboratoire.

Mais ce travail eut des conséquences positives, qui furent plus importantes que l'échec, car il proposait une méthode rigoureusement scientifique pour l'étude de ce problème médical. Selon U. Cerletti, Grassi et Munaron «ont été les premier à poser le problème étiologique sur une base scientifiquement valable» ${ }^{19}$. Ils associent le laboratoire aux recherches épidémiologiques sur le terrain, ils utilisent des modèles animaux, des expériences de contrôle, les procédés de laboratoire qui avaient été mis au point par la physiologie expérimentale et la bactériologie. On peut considérer tout cela, et l'on y reviendra plus loin, comme une conséquence immédiate de la diffusion du modèle explicatif de la microbiologie.

\section{La recherche en Suisse}

En Suisse également, au début du XX $\mathrm{X}^{\mathrm{e}}$ siècle, les recherches sur l'étiologie du goitre sont dominées par la recherche d'un «agent causal», d'un microbe responsable de la maladie. Un programme placé sous la direction de W. Kolle et lancé en 1907 par un comité national suisse avait pour objectif l'isolement d'un «facteur causal». H. Bircher avait proposé en 1883 une théorie selon laquelle le goitre serait une maladie infectieuse chronique et Kolle avait émis l'hypothèse de la présence de parasites intestinaux «toxigéniques». Mais, à part la distribution géographique de l'endémie, qui semblait suggérer une infection lente, on ne parvint pas à établir, en laboratoire, la démonstration des phénomènes infectieux. Toutes les recherches bactériologiques visant à 
isoler des microbes dans le sang et les organes des malades donnaient des résultats négatifs. On ne trouvait jamais ni antigènes ni anticorps.

Dans ses conclusions, présentées au Congrès médical suisse de 1909, Kolle finit par soutenir la théorie du terrain, liant l'origine de la maladie à l'eau tirée d'une structure géologique particulière. La seule mesure prophylactique proposée, c'est de faire bouillir l'eau, pour la priver d'agents toxiques éventuels. Le principe de base est toujours celui d'un agent positif, actif, même si l'idée d'une contagion vivante est abandonnée et substituée par l'expression beaucoup plus neutre de «noxa du goitre».

\section{Les conséquences positives du modèle microbiologique}

Plusieurs historiens ont évoqué les conséquences négatives de la diffusion explosive de la microbiologie, en particulier la tendance à attribuer à un microbe la responsabilité de toute maladie ${ }^{20}$. D'autre part, comme Carter ${ }^{21}$ l'a déjà suggéré, le développement de la théorie des germes, au lieu d'être un obstacle pour l'étude des maladies de carence, a déterminé un changement d'attitude théorique en médecine et en hygiène. Il faut ajouter que l'impact de la bactériologie se réalisa surtout dans la pratique, grâce aux succès thérapeutiques obtenus, qui vont devenir, il est permis de dire pour la première fois sous cette forme, un critère efficace et discriminant pour l'établissement d'une étiologie morbide. La diffusion du modèle explicatif issu de la bactériologie produisit donc des effets positifs qui furent finalement d'une importance beaucoup plus grande que le temps perdu sur des voies de recherche sans issue. Ces effets furent aussi bien pratiques, concernant la thérapie, l'hygiène, la prophylaxie des maladies endémiques et épidémiques, que théoriques, grâce à l'affirmation d'un nouveau critère de scientificité en médecine, fondé sur une définition originale de la causalité et de la spécificité et sur le rôle décisif de l'expérimentation et des modèles animaux.

Les débats sur la spécificité des agents morbides à la fin du XIX ${ }^{\mathrm{e}}$ siècle permirent la diffusion d'un idéal expérimental dans l'ensemble de la médecine. Par conséquent les médecins et les responsables de l'hygiène et de la santé publique furent induits à réexaminer l'ensemble des données connues pour ne faire confiance qu'aux résultats de première main et confirmés par le laboratoire. Les résultats du laboratoire, en s'associant directement à l'observation clinique, donnèrent des significations nouvelles aux données statis- 
tiques de l'ancienne épidémiologie, qui n'avait auparavant d'autre but que montrer sur les cartes géographiques la distribution souvent erratique des épidémies et des foyers endémiques.

Après l'essor de la microbiologie, l'objectif d'une recherche devient d'isoler une cause et non plus de montrer une coexistence constante entre divers phénomènes. Comme Emile Roux l'écrivait, commentant une liste de ses travaux scientifiques,

«la bactériologie définit la maladie par sa cause, et nous permet de différencier la diphtérie vraie des angines à fausses membranes dues à une tout autre cause».

Les postulats de Henle-Klebs-Koch, sur lesquels se construit la portée scientifique de la nouvelle discipline, visent une cause unique, spécifique et discriminante pour chaque maladie ${ }^{22}$. Sans ces postulats, une coïncidence absolue ne pouvait pas être considérée une preuve de causalité spécifique. Avec les postulats, le médecin de laboratoire peut finalement opérer une distinction claire entre facteurs et cofacteurs, comme en biochimie, et ce n'est pas un hasard si la théorie des fermentations est à l'origine aussi bien de la microbiologie que de la biochimie. Il n'y a plus de causes différentes au même niveau épistémologique, mais on peut établir une hiérarchie de causes, avec une seule cause déterminante et d'autres facteurs considérés comme accessoires, même s'ils sont nécessaires et responsables de la variabilité de la réponse à la cause efficiente (l'hérédité, l'alimentation, les facteurs psychosomatiques, les conditions du milieu, etc.).

Après une première période de rigidité, les postulats de Henle-KlebsKoch, appliqués à la détermination de la nature de l'agent causal, furent élargis pour prendre en compte aussi les circonstances de l'infection et l'influence de la réponse individuelle, surtout au niveau des manifestations cliniques de la maladie. Cela permit d'appliquer cette méthode d'analyse étiologique aux maladies chroniques et aux endémies à lente diffusion, par l'établissement d'un ordre temporel correct dans l'apparition de l'agent causal et de la maladie, ainsi que par la détermination de la spécificité de l'effet de l'agent causal pour le développement de la maladie.

Dans la plupart des cas, une maladie est le résultat d'une chaîne causale qui lie la cause spécifique et les cofacteurs au milieu et à la susceptibilité individuelle. L'existence de cette chaîne donne la possibilité de lutter efficacement contre une épidémie ou une endémie. En pratique, afin d'interrompre la transmission d'une épidémie ou d'une endémie, on peut éliminer un facteur nécessaire de la chaîne, même si celui-ci n'est pas la cause unique de 
la maladie. Par exemple, dans le cas du paludisme, le vecteur ne peut pas être considéré la cause spécifique de la maladie (rôle qui doit être assigné aux plasmodiums). Toutefois, le passage par le vecteur est indispensable pour le cycle de vie du parasite; par conséquent, en éliminant les moustiques, on éliminera le paludisme, interrompant le cycle de vie du parasite. L'éradication du vecteur fut donc posée comme objectif prioritaire de la lutte contre le paludisme, dans la conviction que l'éradication du vecteur était indispensable pour l'éradication de la maladie. Conviction erronée, car la simple diminution de la densité des populations d'anophèles au dessous d'un seuil critique, déterminé par les conditions climatiques et les caractères biologiques du vecteur et du parasite, se révéla souvent suffisante pour interrompre la transmission de la maladie.

En ce qui concerne le goitre et le crétinisme, le principe fondamental était l'établissement de la spécificité d'un effet ou d'un facteur. Mais trois obstacles se présentèrent à l'élaboration d'un modèle étiologique de l'endémie: 1) la difficulté de mesurer cet effet quantitativement, à cause de l'extrême variabilité des manifestations cliniques et des diversités géographiques;2) la difficulté de sélectionner témoins pour vérifier la spécificité de l'agent causal; 3) la difficulté d'établir correctement la séquence temporelle des événements et des manifestations cliniques.

Sur la base des vastes connaissances actuelles, l'analyse du médecin et de l'historien de la médecine permet d'expliquer les difficultés liées à la démonstration d'un modèle étiologique, car la même pathologie ou le même état clinique peut être produit par des agents étiologiques différents; en effet, une cause spécifique nécessite souvent plusieurs cofacteurs qui doivent agir en même temps pour produire la maladie et qui peuvent cacher la causalité spécifique. Enfin, l'agent causal peut avoir des effets cliniques variables dans différentes aires géographiques ou selon les variabilités individuelles, et donner origine à un gradient de réponses, liées elles aussi à la constitution génétique, à l'état immunologique des individus et des populations, au niveau socio-économique, aux comportements individuels et sociaux.

On peut comprendre, dès lors, l'impossibilité d'établir une étiologie sûre pour le goitre et le crétinisme endémiques, sujet encore aujourd'hui très débattu, même après les grands succès obtenus par la prophylaxie iodique. En effet, ce succès a été longtemps considéré comme un indice de la causalité de la maladie. Si l'élimination de la carence d'iode peut supprimer le goitre, en déplaçant le critère d'efficacité de la thérapie vers la prophylaxie, on 
arrive à la conclusion que cette carence doit nécessairement être considérée comme la cause de la maladie.

Néanmoins, cette conclusion a été souvent rejetée. Par exemple, I. Greenwald considère que cette efficacité, amplifiée par les producteurs d'iode, a joué un rôle négatif dans l'établissement de la vraie étiologie du goitre, en limitant le regard des chercheurs «pendant les quarante dernières années». Cet auteur soutient, au contraire, l'hypothèse de la nature infectieuse de l'agent causal, car les connaissances sur l'histoire du goitre et sur sa diffusion dans l'espace et dans le temps et la variabilité de ses manifestations cliniques sont incompatibles avec toutes les théories existantes sur la nature de la maladie, sauf celle d'une infection caractérisée par différentes souches dans les différentes régions et les différentes périodes historiques. La situation, c'est la conclusion que Greenwald tire de son analyse, «est tout à fait semblable à celle de la tuberculose au XIX ${ }^{\mathrm{e}}$ siècle. L'hérédité joue un rôle important dans la détermination de la résistance ou de la susceptibilité individuelle, comme le régime. Mais la maladie est infectieuse ${ }^{23} . »$

\section{Les conséquences du pastorisme pour la prophylaxie}

C'est surtout au niveau de la prophylaxie que le pastorisme a produit les conséquences les plus importantes, par la redéfinition même de la thérapie, au moyen de laquelle on va désormais prévenir au lieu de guérir ${ }^{24}$.

Tout au long du $\mathrm{XIX}^{\mathrm{e}}$ siècle, à partir des données épidémiologiques, le goitre et le crétinisme étaient devenus, surtout en Suisse et en Italie, l'un des terrains d'élection pour le mouvement hygiéniste. Toutefois, la doctrine des hygiénistes pré-pastoriens n'est marquée par aucun argument central, par aucune idée-guide. Elle est faite d'une accumulation de connaissances détaillées, non interprétées par un modèle théorique général, d'une agglomération de statistiques, d'études de cas, d'anecdotes, accompagnée sur le plan pratique par des conseils, des précautions, des recettes, des règlements. Si la maladie n'a pas une cause spécifique, unique, mais peut être causée par des facteurs multiples (le sol, l'eau, l'air, la constitution, l'hérédité, les déchets, les odeurs, la saleté, la pauvreté, la sexualité, les aliments, l'urbanisme, l'éducation, même le manque de force d'esprit et de foi), il ne faut rien négliger, il faut tout considérer. Si beaucoup de causes se mêlent, aucune position tranchée n'est permise. La variété des causes possibles impose la nécessité de les combattre par des moyens également variés et nombreux. 
Aucune cause n'est vraiment sûre et par conséquent aucune ne peut vraiment être considérée comme secondaire et mise de côté. Le mouvement hygiéniste était donc dans une situation paradoxale: d'un côté c'était un mouvement social très répandu qui voulait tout prendre en charge, et de l'autre il proposait une série de mesures coûteuses et difficiles à réaliser, car même les programmes les plus simples pouvaient être trahis par des événements qu'on ne pouvait ni dominer ni même comprendre. Dans ces conditions, même si on avait des résultats importants, comme par l'approvisionnement d'eau pure afin de prévenir le choléra, il était souvent difficile de convaincre les pouvoirs publics d'investir pendant des décennies les sommes énormes nécessaires à des vastes programmes hygiéniques. Aux yeux des hygiénistes, des réformateurs sanitaires, des législateurs, l'essor de la microbiologie marque le passage à la certitude des causes et des actions prophylactiques. Les hygiénistes prennent de l'assurance avec les succès thérapeutiques de la bactériologie, et ils deviennent convaincants même quand ils s'intéressent à des endémies qu'on ne considérait pas de nature infectieuse, et qui donc n'appartenaient pas strictement au domaine de la bactériologie. Le modèle microbiologique, fondé sur un rapport continu entre la clinique, l'épidémiologie et le laboratoire, permit une concentration des efforts et une efficacité nouvelle des politiques de santé publique.

La simple définition d'un acteur suffit à «faire croire» en un programme de recherches nouveau et aux mesures prophylactiques relatives. Le microbe est un outil théorique qui produit une nouvelle définition de la causalité en médecine, mais c'est aussi un moyen d'action. La politique sanitaire ne vise plus la totalité, mais intervient avec une grande sûreté sur un «facteur» devenu non seulement visible, mais bien identifié et classifié, spécifique. Isoler une seule cause possible permet de faire des prédictions et donc de construire un programme d'action de longue durée. C'est cela qui accorde finalement une fiabilité aux programmes d'hygiène publique et de prophylaxie au niveau national et international.

\section{La prophylaxie iodique}

Après la première guerre mondiale, qui démontre spectacularement l'efficacité de la prévention et de la prophylaxie, on établit des programmes sanitaires pour un grand nombre de maladies endémiques et infectieuses. 
Pour ce qui concerne le goitre et le crétinisme, on introduit la prophylaxie iodique sur une vaste échelle dans la plupart des pays concernés.

Aux Etats-Unis, D. Marine (1880-1976) et O.P. Kimball, en se fondant sur des résultats expérimentaux obtenus sur les truites, introduisent la prophylaxie iodique dans les écoles en $1917^{25}$, même si Marine n’a jamais affirmé qu'une insuffisance de iode était la seule cause du goitre.

En Suisse, Heinrich Hunziker, en 1915, refuse la théorie de l'infection et soutient que le goitre est le résultat d'une tentative d'adaptation de l'organisme à un régime pauvre en iode et que par conséquent

«tout accroissement de la thyroïde peut être prévenu en donnant à la population la même quantité d'iode qu'elle doit arracher à la nature par l'accroissement de la thyroïde» ${ }^{26}$.

Un rapport de Friedrich de Quervain présenté le 24 juin 1922 à la Commission suisse pour le goitre propose l'introduction de la prophylaxie iodique sur des bases purement empiriques, jusqu'à ce que «le progrès des recherches permettra de remplacer la prophylaxie empirique par une prophylaxie fondée sur l'étiologie» ${ }^{27}$. Th. von Fellenberg, du Bureau fédéral de la santé à Berne, réalise une enquête rigoureuse sur la présence d'iode en Suisse, produisant un tableau complet du cycle de cet élément dans la nature. Ce travail va constituer la base scientifique de la prophylaxie iodique en Suisse ${ }^{28}$. Introduite graduellement, canton par canton, à partir de 1922, cette prophylaxie rencontre de nombreuses difficultés à cause de la distribution insuffisante de sel traité et de la concentration trop basse de l'iode dans le sel, mais elle finit par obtenir un net succès.

En Italie, une association avait réussi à obtenir de la Direction générale de la santé publique la réalisation d'une enquête épidémiologique et statistique soignée ${ }^{29}$. Cette enquête, réalisée entre 1912 et 1914 avec l'envoi de fiches détaillées à chaque «medico condotto», l'officier de santé publique présent dans chaque commune, permit l'élaboration d'un programme prophylactique et thérapeutique, fondé sur quatre principes: 1. la prophylaxie iodique des enfants des régions où le goitre et le crétinisme étaient endémiques; 2 . le traitement de la maladie par des préparations iodiques et thyroïdiennes; 3 . la prévention du crétinisme et du goitre par administration des mêmes préparations; 4. «l'éloignement temporaire» des enfants des individus goitreux et des enfants crétins durant la période de croissance. La prophylaxie par le sel alimentaire iodé fut introduite en 1926 dans la Valteline, mais le déclin du goitre et du crétinisme avait déjà commencé plus tôt par l'effet de l'application de ce programme. L'analyse du pourcentage 
des jeunes hommes réformés au recrutement militaire à cause du crétinisme et du goitre dans la même vallée baisse de $15,96 \%$ pour la période 1905-1909 à $1,86 \%$ pour la période 1920-1924. On retrouve les mêmes données dans d'autres vallées alpines, comme par exemple la Valle del Mera (Sondrio).

\section{Conclusions}

Le manque de connaissances détaillées sur l'étiologie du goitre et du crétinisme n'empêcha pas l'efficacité des mesures de prophylaxie dans tous les pays où elles furent mises en pratique. Cette confiance dans la valeur pratique et l'efficacité de la prophylaxie peut être considérée comme un apport positif et important de la révolution pastorienne à l'amélioration de la santé des populations. Le médecin n'a pas besoin de la certitude philosophique pour agir; il lui suffit d'avoir confiance dans l'efficacité thérapeutique d'un traitement ou d'une mesure prophylactiques. Le modèle pastorien donna aux médecins la force de se convaincre et de convaincre les pouvoirs politiques que le goitre et le crétinisme pouvaient être vaincus par de simples mesures de prophylaxie généralisée. 


\section{Notes et bibliographie}

1 Brouardel, P., Allocution au Congrès International d'hygiène de Paris, 1889, cité par Vallery-Radot, R., La vie de Pasteur, Paris, Hachette, 1922, pp.660-661.

2 «Nous pensons - écrivait le Concours Médical en 1881 (29-1) - que, malgré les attaques un peu passionnées de Monsieur Pasteur, la clinique n'est pas encore tout à fait morte.»

3 Sur les réactions des journaux médicaux à la révolution pastorienne voir: Latour, B., Le théâtre de la preuve, in Salomon-Bayet, C. (réd.), Pasteur et la révolution pastorienne, Paris, Payot, 1986, p. 335-384.

4 Salomon-Bayet, C. (réd.), Pasteur et la révolution pastorienne, Paris, Payot, 1986, p. 17.

5 Voyages dans les Alpes (t. II, 1786) par H. B. de Saussure (1740-1799); Observations faites dans les Pyrénées (1789), par Raymond de Carbonnière (1755-1827); Sui gozzi e sulla stupidità dei cretini che in alcuni paesi l'accompagna (1789), par Vincenzo Malacarne (17441816); Traité du goitre et du crétinisme (1799) par F. E. Fodéré (1764-1835).

6 Sur l'histoire du goitre et du crétinisme, voir: Medvei, V.C., A History of endocrinology, Lancaster, MIT Press, 1982; AA.VV., Endemic Goiter, Washington, P.A.H.O., 1969; Lancer, P., History of goitre, in Endemic Goiter, W.H.O. Monograph Series, Genève, W. H. O., 1960, 9-25; Merke, F., Geschichte und Ikonographie des endemischen Kropfes und Kretinismus, Bern, Hans Huber, 1971, Trad. anglaise, History and iconography of endemic goiter and cretinism, Bern, Hans Huber, 1984, Pazzini, A., Storia del gozzo, Roma, Istituto di Storia della Medicina, 1957.

7 L'extirpation chirurgicale du goitre avait longtemps été un insuccès, et les échecs renouvelés avaient persuadé l'Académie de Médecine de Paris à se prononcer avec détermination contre cette technique. Mais par l'effet de l'introduction de l'anesthésie générale en 1846, de l'antisepsie en 1867, des premières pinces hémostatiques, et done de l'hémostasie, en 1875, la chirurgie du goitre connut une véritable évolution. Theodor Kocher (1841-1917) à Berne et Jacques-Louis Reverdin (1842-1929) à Genève obtiennent dès lors des résultats thérapeutiques positifs par l'ablation totale ou partielle de goitres, dans des conditions de grande sécurité opératoire. En 1889 Kocher en était à sa $250^{\circ}$ extirpation; en 1895, à la $1000^{\circ}$. cf. Bornhauser, S., Zur Geschichte der Schilddrüsen- und Kropfforschung im 19. Jahrhundert, Aarau, Sauerländer, 1951 (Veröff. Schweiz. Ges. Gesch. Med. u. Naturwiss. 19).

8 Coindet, J.-F., Mémoire sur la découverte d'un nouveau remède contre le goitre, in Bibliothèque universelle de Genève 14, 1820, p. 190-198; Nouvelles recherches sur les effets de l'iode et sur les précautions à suivre dans le traitement du goitre avec ce nouveau remède, in Bibl. univers. Genève 16, 1821, p. 140-152.

9 Article 'lode' dans Ure, A., Dictionnaire de chimie, (1821) trad. fr. 1823, Paris, Leblanc, t. III, pp.419-437. cf. Canguilhem, G., Etudes d'histoire et philosophie des sciences, Paris, Vrin, 1975, p. 283-4.

10 Ce syndrome fut étudié d'une façon systématique par F. Rilliet (1814-1861): Mémoire sur l'iodisme constitutionnel, in Bull. Acad. Méd. Paris 25, 1859, p. 382.

11 «Je considère comme certain que le goitre disparaitrait des Cordillères si l'autorité prenait des mesures convenables pour qu'il soit établi dans chaque chef-lieu de canton où le goitre est endémique un dépôt de sel contenant de l'iode et dans lequel chaque habitant pourrait aller acheter le sel nécessaire à sa consommation.» (Annales de chimie et de physique, 40, 1825 , p. 163). 
12 Grange, J.-J., Sur les causes du goitre et du crétinisme et sur les moyens d'en préserver les populations, in Gaz. Méd. Paris, 19, 1851, p. 275.

13 Chatin, G. A., Présence de l'iode dans les plantes d'eau douce. Conséquences de ce fait pour la géologie, la physiologie végétale, la thérapeutique et peut-être pour l'industrie, in C.R. Acad. Sciences 30, 1850, p. 352-54; Un fait dans la question du goitre et du crétinisme, in C.R. Acad. Sciences 36, 1852, p. 652.

14 Virchow, R., Die krankhaften Geschwülste, Berlin, Hirschwald, Bd.III, 1867, p. 59.

15 Carpenter, K. J., The History of Scurvy and Vitamin C, Cambridge, Cambridge University Press, 1986; McCollum, E. V., A History of Nutrition, Boston, Houghton Mifflin Co, 1957.

16 Follis Jr., R. H., Cellular pathology and the development of the deficiency disease concept, in J. Hist. Med., 34, 1960, p. 291-317.

17 Grassi, G. B., Munaron, L., Ricerche preliminari dirette a precisare la causa del gozzismo e del cretinismo endemici, in Rend. R. Accad. Lincei 13, 1903-1905; Uno sguardo alle nostre ricerche sul gozzo e sul cretinismo endemici, in Rend. R. Accad. Lincei 14, 1905; Grassi, G.B., Sulla etiologia del gozzismo, Roma, Tipografia del Senato, 1914.

18 Grassi considère qu'il y avait quatre endémies typiques de l'Italie: la malaria, la pellagre, le goitre et le crétinisme. Les deux premières avaient été vaincues à la fin du XIX ${ }^{e}$ siècle, laissant «al secolo attuale l'incarico di ricercare la causa del gozzo e del cretinismo [...] Questo mistero ci ha attratti, l'utile che dallo svelarlo poteva derivare alle popolazioni ci ha spinti a dedicare a questo compito la nostra opera indefessa».

19 Cerletti, U., Il cretinismo endemico, in Funzioni e disfunzioni tiroidee, Lezioni tenute dai Proff. Castaldi, Righini, Cerletti, Rossi, Lugano. Milano, Istituto Sieroterapico Milanese, 1923, p. 73-100; Gozzo e cretinismo, in Arch. Sci. Med. 105, 1957, p. 91.

20 Ihde, A., Becker, S., Conflict and concept in early vitamin studies, in J. Hist. Biol. 4, 1971, p. 1-33.

21 Carter, K. C., The germ theory, beriberi, and the deficiency theory of disease, in Med. Hist. 21, 1977, p. 119-136.

22 Carter, K.C., Koch's postulates in relation to the work of Jacob Henle and Edwin Klebs, in Med. Hist. 29, 1985, p. 353-374.

23 Greenwald, I., Endemic goiter: heredity, deficiency, intoxication or infection?, in Clinical Endocrinology 1, 1960, p. 123-132.

24 «L'hygiène de Pasteur», écrit la Revue Scientifique en 1882, «permet de prévenir les causes morbides, d'éloigner les maladies, pour ne pas avoir à les guérir.»

25 Marine, D., Further observations and experiments on goiter (so called thyroid carcinoma) in brook trout, in J. Exp. Med. 19, 1914. Marine, D., Kimball, O.P., The prevention of simple goiter in man, in J. Lab. Clin. Res. 3, 1917, p. 40-49, Reprint in Nutr. Rev. 33, 1975, p. 272-275: «Simple goiter is the easiest of all known diseases to prevent. [...] It may be excluded from the list of human diseases as soon as society determines to make the effort.»

26 Hunziker, H., Der Kropf, eine Anpassung an jodarme Nahrung, Bern, A. Franke, 1915. Cf. aussi Die Prophylaxe der grossen Schilddrüse, Bern, 1924.

27 de Quervain, F., Schilddrüse und Jod mit Rücksicht auf die Kropfprophylaxe, in Schweiz. med. Wochenschr. 52, 1922, n. 35, p. 857-862.p. 857.

28 Fellenberg, Th. von, Das Vorkommen, der Kreislauf und der Stoffwechsel des Jods, in Ergebnisse der Physiologie 25, 1926, p, 176-363.

29 AA. VV., Conferenza sulla erigenda istituzione per lo studio, la profilassi e la cura del gozzo e del cretinismo endemici, Roma, Tipografia Cooperativa Sociale, 1909. 


\section{Summary}

\section{The bacteriological revolution and the theories on the aetiology of goiter and cretinism}

During the 19th century, a large number of researches produced many different and even contradictory theories about the aetiology of endemic goiter and cretinism. The bacteriological revolution, i. e. the acceptance of the germ theory of infectious diseases, pushed scientists to try to isolate a germ for every disease. In the case of goiter and cretinism, this was fruitless endeavour. Nevertheless, the impact of the bateriological revolution was largely positive because it provided a new scientific model of the genesis of disease and strengthened confidence in the possibility of fighting endemic as well as epidemic disease by simple but generally applied preventive measures such as, in the present case, iodine prophylaxis.

\section{Zusammenfassung}

Die bakteriologische Revolution und die Theorien über die Ätiologie von Kropf und Kretinismus

Die Forschungen des 19. Jahrhunderts liessen viele Theorien über die Ursachen des endemischen Kropfs und des Kretinismus entstehen. Die bakteriologische Revolution, d. h. die Anerkennung der Keimtheorie der Infektionskrankheiten durch Ärzte und Naturwissenschaftler, gab den Ansporn, für alle Krankheiten einen bakteriellen Erreger zu suchen, was sich im Falle von Kropf und Kretinismus als Irrweg erwies. Dass man ihn beschritt, wurde durch das Fehlen des Begriffes der Mangelkrankheiten begünstigt, der erst zu Beginn des 20. Jahrhunderts entwickelt wurde. Trotzdem hat sich die bakteriologische Revolution im medizinischen Denken letztlich auch auf diesem Gebiet günstig ausgewirkt: sie vermittelte ein neues wissenschaftliches Modell der Krankheitsentstehung und stärkte das Vertrauen in die Möglichkeit, endemische wie epidemische Krankheiten durch allgemein angewandte, prophylaktische Massnahmen einfacher Art - im gegebenen Fall die Jodprophylaxe - wirksam bekämpfen zu können.

Professeur Bernardino Fantini

Institut Louis Jeantet d'histoire de la médecine

Centre médical universitaire

CH-1211 Genève 4 